\title{
The Immune Contexture and Cancer Therapy Aspects of the TIM- 3 Checkpoint Pathway
}

\author{
Yunxuan $\mathrm{Xie}^{1 *}$ \\ ${ }^{1}$ Department of Chemical \& Biomolecular Engineering, Johns Hopkins University, Baltimore, Maryland 21218, United States
}

\begin{abstract}
The advent of immune checkpoint therapy revolutionizes the oncology field by achieving durable anti-tumor efficacy and immense clinical progress. Among the immune checkpoints, T cell immunoglobulin and mucin domain 3 (TIM-3) has been reported to express on both innate and adaptive immune cells where it accounts for immune tolerance and tumor aggression. It shows promising effects that are applicable in several cancer types. This review provides a broad overview of the main TIM-3 relative signaling pathways and its immune regulatory role to shape cancer development positively. The importance of TIM-3 inhibitors in cancer control were also summarized according to the latest reports, discussing its clinical trial data that inform the rationale for TIM-3 blockade.
\end{abstract}

\section{Introduction}

Cancer refers to one of the world's largest health problems characterized by the development of abnormal somatic cells, which results from genetic or epigenetic alternations that divide uncontrollably and have the ability to infiltrate and destroy normal body tissue[1]. The historical battle with cancer has developed the standard of care therapeutic interventions such as chemotherapy, surgery and radiation. It's not until last century that medical sciences start to manipulate the immune system to fight cancer, which marks the establishment of immune-mediated therapies[2].

Immunotherapy has been a revolutionary treatment for its potential to eliminate types of cancer and their metastases while presents low side effect compared with the chemotherapy or radiotherapy. Strategies of immunotherapy mainly involve cytokines therapy, oncolytic viruses, cancer vaccines, cell-based therapies and immune checkpoint inhibitors[3]. Though the prevailing PD-1, CTLA-4 immune checkpoint targeting therapies show compelling antitumor activity in several types of cancer, the resistance of these therapies has been increasingly observed in clinical trial such as colorectal cancer. Thus, the need for exploring other co-inhibitory factors is urgent to improve current immunotherapy.

T cell immunoglobulin and mucin domain 3 (TIM-3), a type I trans-membrane protein, showed up as a promising immunotherapy target and it has been recognized as a pivotal member in TIM family, which is composed of three members in humans including TIM-1, TIM-3 and TIM-4[4]. TIM-1 is preferentially expressed on Th2 (T helper type 2) cells and functions as potent costimulatory molecule for T-cell activation, while TIM-
4 is solely expressed on APCs as it mediates the phagocytosis of apoptotic cells. TIM-3 marks the exhaustion of Th1 (T helper type 1) cells and enhances regulatory function of FoxP3+Tregs. The reported TIM-3 expression on monocytes, dendritic cells and nature killer (NK) cells also exhibit inhibitory role in innate immune responses[4]. As a result, TIM-3 mediates negative immune regulatory response associated with $\mathrm{T}$ cell exhaustion and suppression of innate immune system[4].

Herein, we primarily focus on what is known about the regulatory mechanisms of TIM-3 in the context of tumor immunity and autoimmunity. We further summarize its expression and function on different cell types and how the crosstalk regulates immune response and disease progression. A better understanding of the clinical trials will also shed the light on the TIM-3 blockade in future cancer immunotherapy.

\section{TIM-3 Relevant Signaling Pathway}

The endogenous ligands of TIM-3 have been identified as Galectin-9, Carcinoembryonic antigen cell adhesion molecule like I (Ceacam1), high-mobility group box 1(HMGB1) and phosphatidylserine. All of these ligands have been widely researched to delineate the TIM-3 mechanism of action under the context of immune response.

\subsection{Galectin-9}

Galectin-9, a $\beta$-galactoside mammalian lectin protein, expresses on various tissues, including liver, small intestine, thymus, spleen, lymph nodes etc[5]. Its carbohydrate-binding domain specifically recognizes the TIM-3 IgV domain, which leads to the inhibition of

*Corresponding author's e-mail: 1dico930@163.com 
immune system. In the adaptive immune response, the CD4+T cells differentiate into Th1 and Th2 cells after intracellular and extracellular pathogens activation[6]. TIM-3 specifically expresses on differentiated Th1 cells, which acts as a trafficker for galectin-9 interaction (Figure 1), they evolved to selectively eliminate TIM-3 positive Th1 cells and thus dampen Th1 immunity[7]

\subsection{CEACAM1}

Carcinoembryonic antigen cell adhesion molecule like I (CEACAM1), the highly conserved member of carcinoembryonic antigen (CEA) family, is a well-known molecule expressed on $\mathrm{T}$ cells and involves in modulating its function[8]. The structural similarity of N-terminal domains shared by TIM-3 and CEACAM1 enables their heterodimeric interaction in cis and trans configurations, their heterophilic ligation accounts for BAT3 release and $\mathrm{T}$ cell inhibition, which is responsible for the toleranceinducing function of TIM-3[9]. As shown in Figure 1, without TIM-3 ligand participation, Bat-3 (HLA-B associated transcript 3) binds to the cytoplasmic tail of TIM-3, allowing phosphorylation of specific tyrosine residues through Lck, Fyn, which is followed by downstream recruitment of signaling adaptors that promote T-cell proliferation. However, the presence of CEACAM1 supersede Bat-3 and result in its release from TIM-3 tail, leading to Lck inactivation that downregulates TCR proximal signaling and T cell viability[10].

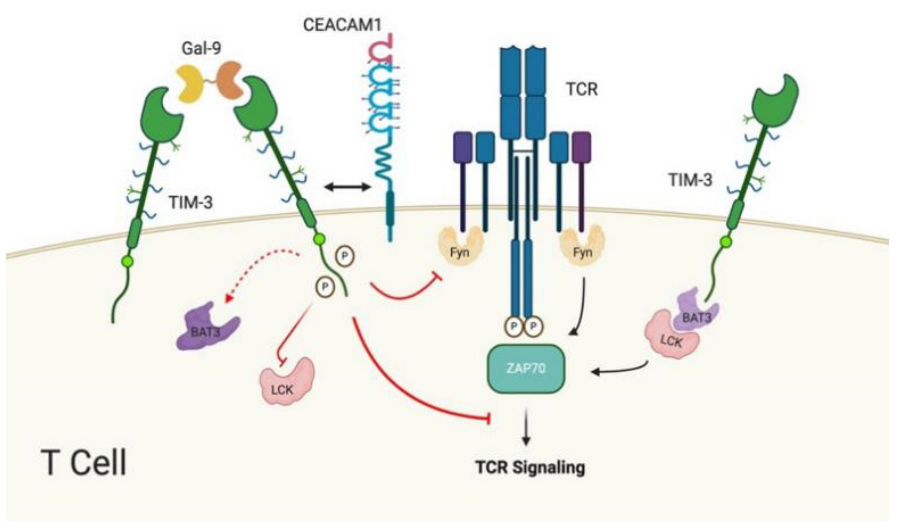

Figure 1. Representative TIM-3 signaling pathways in T cell.

\subsection{HMGB1}

Served as the first line barrier to prevent microbial infection, the innate immune system manipulates germline-encoded pattern recognition receptors (PRRs) to recognize damage-associated molecular patterns (DAMPs), eliciting cellular immune surveillance by activating innate immune cells[11]. As a critical sensor among innate immune cells, the dendritic cells (DCs) actively secrete the HMGB1, one of DAMPs that senses endogenous danger signals, and promote T-cell and B-cell responses. HMGB1 presents nucleic acids into endosome, a key step for DCs to initiate protective immune response. TIM-3 interferes this HMGB1-mediated DNA transport by binding to HMGB1, impairing the PRRs-mediated innate immune response[12].

\subsection{PtdSer}

Differing from other protein ligands of TIM-3, PtdSer is a kind of phospholipid that confined to the inner leaflet of the bilayer[13]. PtdSer transversely goes across the bilayer and redistributes on the surface of apoptotic cells, which is a key signal for provoking phagocytic cellsmediated engulfment[14]. The CC' and FG loops of PtdSer provide the conserved binding pocket for TIM-3. Thus, their interaction plays a hindrance to those regulating for immune response and apoptotic cell clearance. The expression of TIM-3 on DCs, macrophages, and $\mathrm{T}$ cells engages in the PtdSerdependent recognition and phagocytosis of apoptotic cell[15].

\section{TIM-3 Expression and Its Function}

The transcriptional control and expression of TIM-3 on various cell types have been an active research area to delve into the correlation between TIM-3 and malignant tumors. It has been well-documented that the upregulation of TIM-3 on tumor-infiltrating cells is associated with poor prognosis in different cancer settings such as prostate cancer, colon cancer, gastric cancer[4]. Relevant investigations also suggested that the expression of TIM3 on certain innate immune cells acted as a negative regulator to suppress anti-tumor immunity[16]. Besides, the widespread expression of TIM-3 on multiple cancer cells may lead to tumor progression and metastasis.

\subsection{TIM-3 and T cells}

As one of the effector populations that following the activation of CD4+T-helper cells, Th1 cells have been specifically marked by high-level expression of TIM-3 after its polarization. Th1 cytokines IFN- $\gamma$ and IL-12 promote CTL activity and macrophage activation, which plays a pathogenic role in autoimmune disease and allergies. However, the interaction between TIM-3 and its ligands may serve to limit the expansion of Th1 population and lead to inappropriate Th1 response[17]. 
Experimental autoimmune encephalomyelitis (EAE) had been established as an animal model to study Th1 induced autoimmune disease. Administration of anti-TIM-3 fusion protein exacerbated the disease since the interaction between TIM-3 and its ligands was blocked and it would account for Th1 hyperactivation and amplify Th1 cytokines production. Plus, blockade of TIM-3 also abrogated the induction of tolerance in Th1 cells, indicating that TIM-3 may represent as an inhibitory receptor that restrain effector $\mathrm{T}$ cell function[8].

TIM-3 also functions to enforce $\mathrm{CD} 8+\mathrm{T}$ cell exhaustion and dysfunction, a state CD8+T cells become unresponsive to antigens and fail to produce immunestimulatory cytokines[18]. The functional defects in $\mathrm{CD} 8+\mathrm{T}$ cells were initially characterized during chronic lymphocytic choriomeningitis virus (LCMV) infection in mice model. Subsequently, the correlation between CD8+T cells exhaustion and TIM-3 expression on virusspecific $\mathrm{T}$ cells was observed in HIV-1 and $\mathrm{HCV}$ infections[19]. In addition, co-expression of TIM-3 and PD-1 was reported on the tumor-infiltrating lymphocytes (TILs) in mice bearing solid tumors. Dual blockade of TIM-3 and PD-1 pathways led to better reversal of $\mathrm{CD} 8+\mathrm{T}$ cells exhaustion than targeting either pathway alone[20]. Aside from solid tumor, targeting the PD-1 and TIM-3 simultaneously was more effective in preventing $\mathrm{CD} 8+\mathrm{T}$ cells exhaustion than monotherapy in systemic hematopoietic tumor model such as advanced acute myelogenous leukemia (AML), which suggested that TIM-3 may regulate CD8+T cell in cooperation with PD1 during tumor growth[21].

In addition to effector T cells and CD8+T cells, TIM3 expression is also characterized on CD4+regulatory $\mathrm{T}$ cells (Tregs) in multiple human and murine tumors, where the highly suppressive Tregs contribute to the formation of barrier against antitumor immunity. The specific accumulation of TIM-3+Tregs in human hepatocellular carcinoma (HCC) exhibited inhibition of CD8+T cells proliferation and impaired induction of cytokine such as IFN- $\gamma$ and IL-2, which exerted robust immunosuppressive effects in driving antitumor immune response[22]. The TIM-3 expression was also investigated in both CD4+ and CD8+TILs in none small cell lung cancer (NSCLC)[23]. Collectively, the expression of TIM- 3 induces the T cell dysfunction and plays a dispensable role in regulating adaptive immunity.

\subsection{TIM-3 and non-T cells}

Unlike its inducible expression on $\mathrm{T}$ cells, TIM-3 expresses on innate immune cells constitutively. NK eliminates tumor cells induce direct cytotoxicity and take part in the tumor immunosurveillance by producing cytokines Recent studies showed paradoxical conclusions about TIM-3 function in NK cells compared with T cells. In the presence of Gal-9, IFN- $\gamma$ production significantly increased in NK92 cell line with the overexpression of TIM-3. Gal-9-positive acute myelogenous leukemia (AML) also enhanced the IFN- $\gamma$ function of TIM-3+NKcell, which indicated that TIM-3 acted as an activating receptor to mediate NK-cell effector functions[24]. On the contrary, TIM-3 marked the NK-cell exhaustion in advanced melanoma and its expression correlated poor prognosis, which supported the TIM-3 targeting therapy to reverse the exhausted phenotype of NK cells[25].

There's also clinical relevance between TIM-3 expression and myeloid cells. High- level expression of TIM-3 detected on tumor cells and tumor-associated macrophages (TAM) was associated with shorter progression-free survival (PFS) in patient with clear cell renal cell carcinoma (ccRCC)[26]. Furthermore, more precise studies revealed that TIM-3 may promote M2 macrophage polarization that supported the tumorigenesis of colon cancer. These findings suggested that TIM-3 may augment tumor progression by altering macrophage phenotype[27]. Similarly, high expression of TIM-3 also identified on tumor associated DCs (TADCs). DCderived TIM-3 interfered with HMGB1 sensing system to circumvent nucleic-acid mediated stimulation, impeding the immunological response of innate immunity[28].

\section{TIM-3 Inhibitors and Tumor Therapy}

As one of the next generation emerging drugs, different isotypes of antibodies active in the market gives unique biological properties and effector functions. Immune checkpoint antibodies have led to oncological breakthroughs since the debut of anti-PD-1and antiCTLA-4 blockade therapy, which become a great weapon in the fight against several tumor types. Though these classical checkpoint inhibitors demonstrate obvious advantage, there is still an urgent need to unravel novel immuno-checkpoint to get better overall response rates and to eliminate adaptive resistance[29]. TIM-3 could be a promising target because of its widespread expression including T cells, myeloid cells and tumor cells as we discussed above. Increasing numbers of preclinical studies and impressive clinical responses were reported when applied TIM-3 inhibitors monotherapy or combination therapy as shown in Table 1 [10].

Table 1. Clinical development of TIM-3 inhibitors

\begin{tabular}{|c|c|c|c|c|}
\hline Drug & Manufacture/company & Phase & Cancer Type & $\begin{array}{c}\text { ClinicalTrials.gov } \\
\text { identifier }\end{array}$ \\
\hline Sym023 & Symphogen & I & $\begin{array}{c}\text { Metastatic cancer, solid tumor, } \\
\text { lymphoma }\end{array}$ & NCT03489343 \\
\hline
\end{tabular}




\begin{tabular}{|c|c|c|c|c|}
\hline TSR-022 & Tesaro & II & Liver cancer & NCT03680508 \\
\hline MGB453 & Novartis & II & Myelodysplastic syndromes & NCT03946670 \\
\hline $\begin{array}{c}\text { ONO-7807/ } \\
\text { BMS-86258 }\end{array}$ & Bristol-Myers Squibb & II & Solid tumors & NCT03446040 \\
\hline LY3321367 & Eli Lilly and Company & Ia/Ib & Solid tumors & NCT03099109 \\
\hline BGBA425 & BeiGene & I & $\begin{array}{c}\text { Locally advanced or metastatic } \\
\text { solid tumors }\end{array}$ & NCT03708328 \\
\hline R07121661 & Hoffmann-La Roche & I & $\begin{array}{r}\text { Solid tumors, metastatic } \\
\text { melanoma, NSCLC, SLCL, ESCC }\end{array}$ & NCT68 \\
\hline
\end{tabular}

Among the anti-TIM-3 antibodies, R07121661 is a bispecific antibody targeted both PD-1 and TIM-3(Figure 2), which blocks the interaction of TIM-3 with
CEACAM1 expressed on cancer cell and prevents the activation of PD-1with its ligands, enhancing $\mathrm{T}$ cell mediated anti-tumor activity.

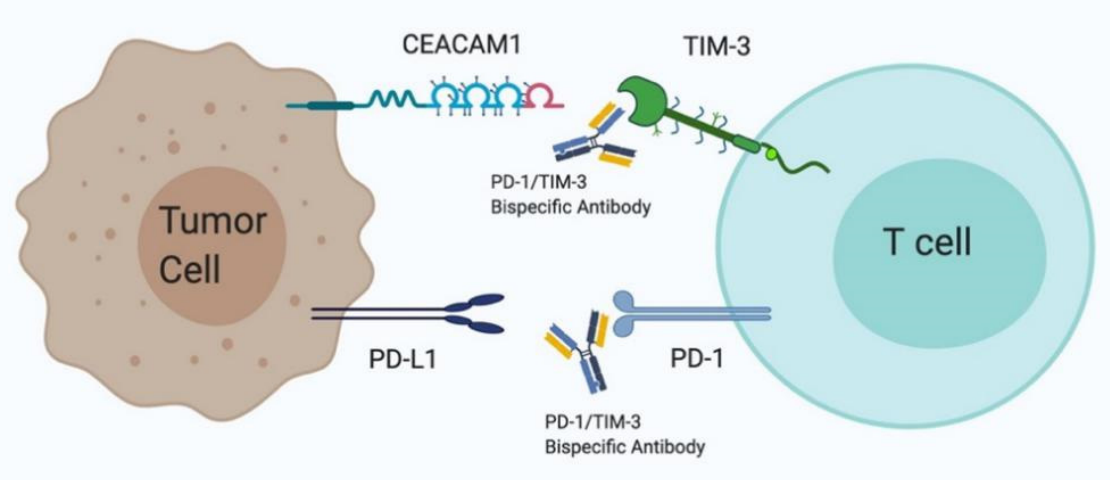

Figure 2. Anti-PD1/TIM-3 bispecific antibody R07121661 mechanism of action.

\section{Conclusion}

Since the expansion of immune checkpoint landscape, TIM-3 has emerged as a novel target for immunotherapy for its relevance to human cancer. Elucidating its interaction with endogenous ligands and relative signaling pathways are critical since it is highly related to the formation of carcinoma and tumor immune evasion. Moreover, the suppressive effects mediated by TIM-3 on both innate and adaptive immune cells underscore the significance of blocking TIM-3 in these cells, by which could convert cell exhaustion state to tumor-reactive state.

As tumor therapies targeting TIM-3 move forward in clinical development, it shows effective therapeutic outcome involving tumor regression and favorable prognosis, particularly in combination of multiple immune parameters, rather than individual ones, might increase prognostic power. Collectively, targeting the TIM-3 pathway has high potential for anti-cancer immunotherapy and provides insights into check-point inhibitors development. Further mechanistic studies and clinical data are warranted to investigate the roles of TIM3 in cancer immunity and therapeutic efficacy of TIM-3 inhibition.

\section{References}

1. Podlaha, O., et al., Evolution of the cancer genome. Trends Genet, 2012. 28(4): p. 155-63.

2. Rius, M. and F. Lyko, Epigenetic cancer therapy: rationales, targets and drugs. Oncogene, 2012. 31(39): p. 4257-65.

3. Farkona, S., E.P. Diamandis, and I.M. Blasutig, Cancer immunotherapy: the beginning of the end of cancer? BMC Med, 2016. 14: p. 73.

4. Das, M., C. Zhu, and V.K. Kuchroo, Tim-3 and its role in regulating anti-tumor immunity. Immunological Reviews, 2017. 276(1): p. 97-111.

5. Wada, J. and Y.S. Kanwar, Identification and characterization of galectin-9, a novel betagalactoside-binding mammalian lectin. J Biol Chem, 1997. 272(9): p. 6078-86. 
6. Luckheeram, R.V., et al., CD4(+)T cells: differentiation and functions. Clin Dev Immunol, 2012. 2012: p. 925135.

7. Yasinska, I.M., et al., The Tim-3-Galectin-9 Pathway and Its Regulatory Mechanisms in Human Breast Cancer. Front Immunol, 2019. 10: p. 1594.

8. Sabatos, C.A., et al., Interaction of Tim-3 and Tim3 ligand regulates $\mathrm{T}$ helper type 1 responses and induction of peripheral tolerance. Nat Immunol, 2003. 4(11): p. 1102-10.

9. Huang, Y.H., et al., CEACAM1 regulates TIM-3mediated tolerance and exhaustion (vol 517, pg 386, 2015). Nature, 2016. 536(7616): p. 359-359.

10. Acharya, N., C. Sabatos-Peyton, and A.C. Anderson, Tim-3 finds its place in the cancer immunotherapy landscape. J Immunother Cancer, 2020. 8(1).

11. Takeuchi, O. and S. Akira, Pattern recognition receptors and inflammation. Cell, 2010. 140(6): p. 805-20.

12. Chiba, S., et al., Tumor-infiltrating DCs suppress nucleic acid-mediated innate immune responses through interactions between the receptor TIM-3 and the alarmin HMGB1. Nat Immunol, 2012. 13(9): p. 832-42.

13. Nagata, S., et al., Exposure of phosphatidylserine on the cell surface. Cell Death Differ, 2016. 23(6): p. 952-61.

14. Verhoven, B., R.A. Schlegel, and P. Williamson, Mechanisms of phosphatidylserine exposure, a phagocyte recognition signal, on apoptotic $\mathrm{T}$ lymphocytes. J Exp Med, 1995. 182(5): p. 1597601.

15. DeKruyff, R.H., et al., T cell/transmembrane, Ig, and mucin-3 allelic variants differentially recognize phosphatidylserine and mediate phagocytosis of apoptotic cells. J Immunol, 2010. 184(4): p. 1918-30.

16. Banerjee, H. and L.P. Kane, Immune regulation by Tim-3. F1000Res, 2018. 7: p. 316.

17. Monney, L., et al., Th1-specific cell surface protein Tim-3 regulates macrophage activation and severity of an autoimmune disease. Nature, 2002. 415(6871): p. 536-41.

18. Gorman, J.V., et al., Tim-3 directly enhances CD8 $\mathrm{T}$ cell responses to acute Listeria monocytogenes infection. J Immunol, 2014. 192(7): p. 3133-42.

19. Jin, H.T., et al., Cooperation of Tim-3 and PD-1 in CD8 T-cell exhaustion during chronic viral infection. Proc Natl Acad Sci U S A, 2010. 107(33): p. 14733-8.

20. Sakuishi, K., et al., Targeting Tim-3 and PD-1 pathways to reverse $\mathrm{T}$ cell exhaustion and restore anti-tumor immunity. J Exp Med, 2010. 207(10): p. 2187-94.
21. Zhou, Q., et al., Coexpression of Tim-3 and PD-1 identifies a CD8+ T-cell exhaustion phenotype in mice with disseminated acute myelogenous leukemia. Blood, 2011. 117(17): p. 4501-10.

22. Yan, J., et al., Tim-3 expression defines regulatory T cells in human tumors. PLoS One, 2013. 8(3): p. e58006.

23. Gao, X., et al., TIM-3 Expression Characterizes Regulatory $\mathrm{T}$ Cells in Tumor Tissues and Is Associated with Lung Cancer Progression. Plos One, 2012. 7(2).

24. Gleason, M.K., et al., Tim-3 is an inducible human natural killer cell receptor that enhances interferon gamma production in response to galectin-9. Blood, 2012. 119(13): p. 3064-3072.

25. Gallois, A., et al., Reversal of NK cell exhaustion in advanced melanoma patients by Tim-3 blockade. Cancer Research, 2014. 74(19).

26. Komohara, Y., et al., The Coordinated Actions of TIM-3 on Cancer and Myeloid Cells in the Regulation of Tumorigenicity and Clinical Prognosis in Clear Cell Renal Cell Carcinomas. Cancer Immunol Res, 2015. 3(9): p. 999-1007.

27. Jiang, X., et al., Tim-3 promotes tumor-promoting M2 macrophage polarization by binding to STAT1 and suppressing the STAT1-miR-155 signaling axis. Oncoimmunology, 2016. 5(9): p. e1211219.

28. Chiba, S., et al., Tumor-infiltrating DCs suppress nucleic acid-mediated innate immune responses through interactions between the receptor TIM-3 and the alarmin HMGB1. Nature Immunology, 2012. 13(9): p. 832-842.

29. He, X. and C. Xu, Immune checkpoint signaling and cancer immunotherapy. Cell Res, 2020. 30(8): p. 660-669. 